\title{
Vibrotactile Perception at Glabrous and Nonglabrous Skin of Fingers: Repeatability of Measurements and Changes Induced by Acute Vibration Exposure
}

\author{
MH Mahbub ${ }^{1}$, Hiroto Ohnari ${ }^{1}$, Kazuko Tanigawa ${ }^{1}$, Kenichi Hiroshige ${ }^{1}$, \\ Yukio TAKAHASHI ${ }^{2}$, Taisuke TogARI ${ }^{1}$ and Noriaki HARADA ${ }^{1}$ \\ ${ }^{1}$ Department of Hygiene, Yamaguchi University Graduate School of Medicine and ${ }^{2}$ National Institute of Occupational \\ Safety and Health, Japan
}

\begin{abstract}
Vibrotactile Perception at Glabrous and Nonglabrous Skin of Fingers: Repeatability of Measurements and Changes Induced by Acute Vibration Exposure: MH MAHBUB, et al. Department of Hygiene, Yamaguchi University Graduate School of Medicine-Objectives: We sought to determine the within-session and between-session repeatability of vibrotactile perception threshold (VPT) measurements and the response patterns in VPT induced by acute exposure to short-term vibration from grasping a vibrating handle, at both glabrous and nonglabrous skin of fingers. Methods: Baseline VPT was recorded twice at glabrous and nonglabrous side of fingers in the right hand of eight healthy volunteers. Then, the subjects were exposed to three exposure conditions (vibration at $31.5 \mathrm{~Hz}$ and $250 \mathrm{~Hz}$, and no vibration), from gripping a vertical handle by the right hand, conducted on 3 different days at an interval of 1-3 wk. After exposure, the subjects released the hand and further VPT measurements at each location were made. Results: Compared to the nonglabrous side, VPT measurements at the glabrous side demonstrated better within-session and between-session repeatability with lower coefficient of repeatability and higher intraclass correlation coefficient. After exposure, a significant increase in VPT was noted under both $31.5 \mathrm{~Hz}$ and $250 \mathrm{~Hz}(p<0.05-$ 0.001 ) exposure conditions in the glabrous finger. In the nonglabrous finger, a pronounced increase in VPT was revealed under $250 \mathrm{~Hz}$ exposure condition ( $p=0.05)$. Conclusions: While measuring VPT at glabrous and/ or nonglabrous fingers, the importance of the site of
\end{abstract}

Received Sep 1, 2010; Accepted Oct 18, 2010

Published online in J-STAGE Nov 25, 2010

Correspondence to: N. Harada, Department of Hygiene, Yamaguchi University Graduate School of Medicine, 1-1-1 Minami-Kogushi, Ube, Yamaguchi 755-8505, Japan

(e-mail:harada@yamaguchi-u.ac.jp) measurement should be considered; the repeatability for such measurements appears to be better at the glabrous site. At high frequency, vibrotactile perception appears to be affected in both glabrous and nonglabrous skin from acute vibration exposure.

(J Occup Health 2011; 53: 10-15)

Key words: Finger vibrotactile perception, Glabrous and nonglabrous skin, Hand-arm vibration, Repeatability

Prolonged use of vibratory hand-held tools can result in the development of a complex and potentially disabling chronic disorder of the upper extremities, known as the Hand-arm Vibration Syndrome (HAVS), which involves vascular, neural and musculoskeletal systems. Neuromuscular impairments in HAVS can lead to a disabling condition that interfere with social and workrelated activities ${ }^{1)}$. A number of research studies has investigated peripheral neuropathy among workers exposed to hand-arm vibration; the use of vibrating power tools can cause a diffuse peripheral neuropathy at multiple sites in the upper extremity ${ }^{2}$.

The measurement of vibrotactile perception threshold (VPT) is currently a commonly used method for the diagnosis of peripheral neuropathy in HAVS, which is characterized by elevated thresholds in peripheral cutaneous perception $^{3)}$. Determination of VPT is also a widely used method to evaluate the peripheral neuropathy related to several other disease states like diabetes, stroke, age-related sensory loss, spinal cord injury etc ${ }^{4,5}$.

Among workers exposed to vibration, quantification of VPT is usually conducted at the glabrous finger (fingertip). However, measurements of VPT have been made widely by different researchers in both laboratory and clinical research works at both glabrous and nonglabrous/hairy 
skin sites across upper and lower extremities including fingers and toes ${ }^{6-9)}$. The determination of VPT is psychophysical in nature as the response to sensory stimulus represents the subjective feeling of vibration sensation by the subject ${ }^{10)}$. Furthermore, though there are striking similarities in the discriminative performance of glabrous and nonglabrous skin, the detection of vibrotactile sense is poorer in nonglabrous skin than in glabrous skin ${ }^{11)}$. These may influence the test-retest repeatability of VPT measurements at glabrous and nonglabrous skin of extremities. To our knowledge, there is a dearth of reported data investigating the within-session and between-session repeatability for VPT measurements at both glabrous and nonglabrous skin. In this study, the purpose was to determine the repeatability (within-session and between-session) of VPT measurements conducted at glabrous and nonglabrous skin of fingers.

Previous research works demonstrated that exposure of subjects to a relatively short duration of hand-arm vibration can significantly affect their digital vibrotactile perception at the glabrous finger/fingertip ${ }^{7,12,13)}$. However, no study has been reported with measurements of cutaneous vibrotactile perception at both glabrous and nonglabrous skin of fingers in subjects with acute exposure to hand-arm vibration. Therefore, another purpose of the present study was to explore the response patterns in cutaneous vibrotactile perception, induced by acute exposure to short-term vibration from grasping a vibrating handle, simultaneously in both glabrous and nonglabrous skin of fingers.

\section{Methods}

\section{Subjects}

Participants in this study were eight healthy nonsmoking normotensive male young volunteers (mean age \pm SE, 24.0 $\pm 0.5 \mathrm{yr}$; mean BMI $\left.\pm \mathrm{SE}, 21.9 \pm 0.6 \mathrm{~kg} / \mathrm{m}^{2}\right)$. None of the subjects was with any known vascular or neurological disease. Written informed consent to participate in this study was obtained from all subjects prior to enrollment. An oral explanation of the test procedure of the study was made to all of them. The subjects were asked to refrain from eating and drinking tea or coffee for at least $2 \mathrm{~h}$ before the beginning of the experimental session and to put on light clothing in the experiment room. The experiment was conducted during the winter season (January-March) approx. at the same time (between 9 am to $5 \mathrm{pm}$ ) on different days for each of the study subjects. The study was approved by the institutional ethics committee of Yamaguchi University School of Medicine.

\section{Experimental design}

All of the participants underwent one trial session on a separate day to familiarize themselves with the experiment protocol. After arrival, all the subjects underwent acclimatization for a period of $30 \mathrm{~min}$ in the temperature- controlled experiment room, seated comfortably on a height adjustable chair without any stress. They positioned their both hands approximately at heart level, palm down over a hard foam padding placed on a wooden table with the left (contralateral) index finger resting near the response button of the hand-switch fixed on the table at a suitable height.

VPT was measured in the right hand rested on a level with the vibrating probe during measurements, over the areas reflecting the cutaneous innervation of three sensory nerves of the hand: median (fingertip of middle finger), ulnar (fingertip of little finger and dorsum of middle phalanx of ring finger) and radial (dorsum of middle phalanx of index finger) nerves. All the subjects had a baseline glabrous and nonglabrous test finger skin temperature of more than $30^{\circ} \mathrm{C}$, which was measured by fixing the sensors of the thermistor to the vibrotactile test sites. At the end of acclimatization period, VPT was measured at each location (one cycle). Measurements on the glabrous (fingertip) and nonglabrous sides were taken with the hand supported and relaxed in pronated and supinated posistions respectively. The subjects placed the finger over a cylindrical vibrating probe of $6 \mathrm{~mm}$ in diameter with a surround (probe-surround gap $2 \mathrm{~mm}$ ) contact force of approximately $2 \mathrm{~N}$. The finger force was displayed on a digital scale of the vibrotactile perception meter. The vibratory stimulus was delivered perpendicular to the skin surface at the frequency of $125 \mathrm{~Hz}$, via a stimulating probe. According to the method referred to as a von Bekesy up/down psychophysical algorithm (ISO13091-1) ${ }^{14)}$, at a constant rate of $3 \mathrm{~dB} / \mathrm{s}$ the vibration magnitude increased until the stimulus was perceived (when the subject was instructed to press the response button by the left index finger) and then decreased until the stimulus could no longer be felt (when the subject was asked to release the response button). This sequence was repeated at least six times. The VPT value was calculated as the mean of the peak (ascending) and trough (descending) thresholds. The four fingers- middle, index, ring and little- were tested in the same order during each cycle of measurements. The baseline VPT measurements were recorded twice at an interval of $5 \mathrm{~min}$ between the two cycles of measurements. The vibrometer display was kept out of the subject's sight.

After the baseline VPT measurements, the subjects were asked to grip the vertical handle by the right hand for $5 \mathrm{~min}$ under a grasping force of approximately $20 \mathrm{~N}$. The grip force produced by the subject was displayed on a strain indicator for visual feedback. Subject's hand was exposed to sinusoidal vibration during this 5 min period $^{15,16)}$. In this experiment, vibration was generated at two frequencies of $31.5 \mathrm{~Hz}$ and $250 \mathrm{~Hz}^{16)}$, with a frequency weighted rms acceleration of $5.5 \mathrm{~m} / \mathrm{s}^{2}$. Then the subject released his hand. After exposure to vibration, three measurements of VPT (after 1, after 2, and after 3) at each location were 
Table 1. Within-session repeatability for VPT measurements

\begin{tabular}{|c|c|c|c|c|}
\hline \multirow[b]{2}{*}{ Session } & \multicolumn{2}{|c|}{ Glabrous finger } & \multicolumn{2}{|c|}{ Nonglabrous finger } \\
\hline & CoR & ICC $(95 \% \mathrm{CI})$ & CoR & ICC (95\% CI) \\
\hline Control & 7.3 & $0.84(0.59-0.94)$ & 10.4 & $0.79(0.50-0.92)$ \\
\hline $31.5 \mathrm{~Hz}$ & 5.4 & $0.91(0.75-0.97)$ & 10.1 & $0.79(0.49-0.92)$ \\
\hline $250 \mathrm{~Hz}$ & 5.8 & $0.87(0.67-0.95)$ & 13.2 & $0.77(0.47-0.92)$ \\
\hline
\end{tabular}

Table 2. Between-session repeatability for VPT measurements

\begin{tabular}{lccccc}
\hline \multirow{2}{*}{ Sessions } & \multicolumn{2}{c}{ Glabrous finger } & & \multicolumn{2}{c}{ Nonglabrous finger } \\
\cline { 2 - 3 } \cline { 6 - 6 } & CoR & ICC $(95 \% \mathrm{CI})$ & & CoR & ICC (95\% CI) \\
\hline Control-31.5 Hz & 6.4 & $0.85(0.62-0.94)$ & & 14.4 & $0.53(0.07-0.81)$ \\
Control-250 Hz & 5.1 & $0.90(0.73-0.96)$ & & 13.3 & $0.69(0.31-0.88)$ \\
$31.5 \mathrm{~Hz}-250 \mathrm{~Hz}$ & 9.0 & $0.69(0.32-0.88)$ & & 11.5 & $0.75(0.43-0.91)$ \\
\hline
\end{tabular}

recorded in a row. Each cycle of measurements took 5 to 6 min.

The control experimental session was identical, but without generation of vibration. Each of the three experimental sessions/conditions (control and vibration at 2 frequencies) was conducted on 3 different days at an interval of 1-3 wk, the sequence of which was chosen in a random order for each of the subjects. All the tests were performed by the same investigator in the same temperature controlled experiment room with an air temperature of 25 $\pm 1{ }^{\circ} \mathrm{C}$; the surface temperature of the handle was also maintained at $25 \pm 0.5^{\circ} \mathrm{C}$. The subjects used ear muffs to shield themselves from the noise of vibration.

\section{Equipments}

A duralumin cylindrical vertical handle (length $15 \mathrm{~cm}$, diameter $4.0 \mathrm{~cm}$ ) with a rigidly fitted pick up on it was mounted on the vibratory plate of an electromagnetic shaker (MES652, Akashi, Japan), which was connected to a computer-controlled power amplifier (S · DA, Akashi, Japan). Vibration level of the duralumin handle was continuously monitored with a vibration meter (VM-20A, Rion, Japan) to ensure a controlled vibration exposure. To display the grip force, a strain gauge connected to a strain indicator (CDV-700A, Kyowa, Japan) was also fixed on the handle. A constant temperature on the surface of the handle was maintained by flowing water of desired temperature, from a water circulation control device (UA100G, Tokyo Rikakikai, Japan) through the columnar space in the middle of the handle. Skin temperature, room temperature and handle temperature were measured by using digital thermistor (SZL-64, Technol seven, Japan) with a measurement accuracy of $\pm 0.15^{\circ} \mathrm{C}$, connected to a scanner (High accurate data logger K730, Technol seven, Japan). VPT was measured by a commercial device
(HVLab Tactile Vibrometer, Institute of Sound and Vibration Research, University of Southampton, UK).

\section{Statistical analyses}

The values of the VPT data were transformed to dB (relative $10^{-6} \mathrm{~ms}^{-2}$ ). To assess within-session repeatability, the baseline measurements performed twice during each of the three sessions/conditions (control, $31.5 \mathrm{~Hz}$ and 250 $\mathrm{Hz}$ ) were used. To determine between-session repeatability, the averaged baseline measurements taken on those 3 different sessions were used. For the repeatability analysis, two measurements were calculated: the coefficient of repeatability $(\mathrm{CoR})^{17)}$ and the intraclass correlation coefficient (ICC) ${ }^{18)}$. Subsequent statistical analyses were performed by using repeated measures ANOVA with Bonferroni correction for multiple comparisons. The VPT data did not show a normal distribution and statistical analyses were performed with data after natural logarithmic transformation and also with non-logarithmic data ( $\mathrm{dB}$ VPT). However, statistical comparisons using both types of data produced similar results and hence, non-logarithmic values (dB VPT) have been shown in the results. The SPSS statistical software version 16.0 was used. Statistical significance was considered at $p<0.05$.

\section{Results}

The CoR and ICC for VPT measurements obtained during a single or different experimental session/s are shown in Table 1.

\section{Within-session repeatability}

The values of CoR were lower at the glabrous site (range, 7.3 to 5.4) than at the nonglabrous site (range, 13.2 to 10.1). The VPT measurements recorded at the glabrous site achieved high ICC (range, 0.91 to 0.84 ) than at the 

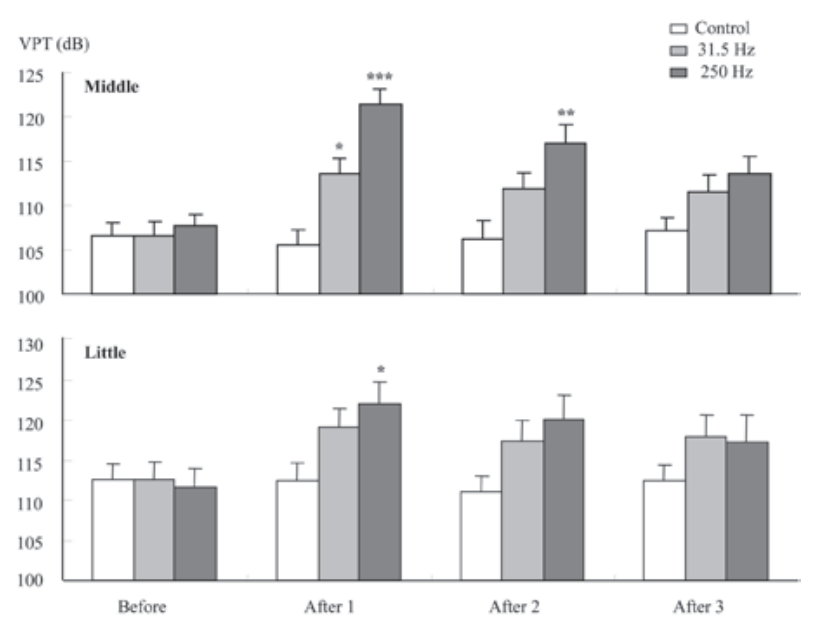

Fig. 1. Mean VPT $(\mathrm{dB})$ in the middle and little fingers (glabrous skin) at different time points under different exposure conditions. Values are mean+SE for eight subjects. Significantly different from the corresponding control values: $* p<0.05, * * p<0.005$ and $* * * p<0.001$.

nonglabrous site with lower ICC (range, 0.79 to 0.77 ).

\section{Between-session repeatability}

The between-session CoR values were also lower at the glabrous site (range, 9.0 to 5.1) than at the nonglabrous side (range, 14.4 to 11.5 ). Overall, the ICC at the glabrous side achieved comparably high values except in one situation (control-250Hz; ICC, 0.69), whereas at the nonglabrous site it achieved lower values (range, 0.75 to 0.53 ).

The averaged baseline VPT including all conditions at the nonglabrous skin was higher compared with the glabrous skin (120.6 dB and $109.7 \mathrm{~dB}$, respectively). Data analysis including only the baseline measurements (before exposure to vibration) showed that the values of VPT obtained during three exposure conditions did not differ significantly in any of the fingers.

\section{Changes in VPT after exposure}

In the middle finger, after exposure, compared to the corresponding control values, the increase in VPT was significant with vibration exposure at $31.5 \mathrm{~Hz}(p<0.05)$ and $250 \mathrm{~Hz}(p<0.001)$ for the first measurement; and at $250 \mathrm{~Hz}(p<0.005)$, for the second measurement (Fig. 1). In the little finger after exposure to vibration at $250 \mathrm{~Hz}$ $(p<0.05)$, the first measurement of VPT was significantly greater than the corresponding control value (Fig. 1). On the other hand in the nonglabrous skin after exposure to vibration, a non-significant general trend toward an increase in VPT was observed relative to the corresponding control values; however in the the ring finger there was a pronounced increase $(p=0.05)$ in VPT under the condition of $250 \mathrm{~Hz}$ for the first measurement (Fig. 2). Overall, in

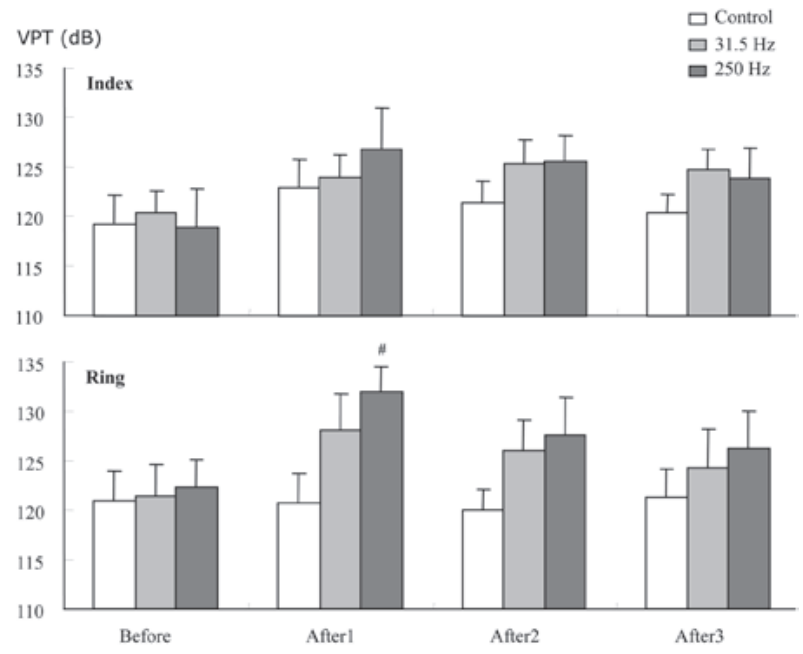

Fig. 2. Mean VPT $(\mathrm{dB})$ in the index and ring fingers (nonglabrous skin) at different time points under different exposure conditions. Values are mean+SE for eight subjects. Significance level of difference from the corresponding control value: ${ }^{*} p=0.05$.

both glabrous and nonglabrous finger, vibration exposure at $250 \mathrm{~Hz}$ produced the larger increase in VPT.

\section{Discussion}

At present, VPT is measured by psychophysical methods as no objective test is available. To determine any true changes in VPT following an intervention, such as exposure to vibration, the within-session repeatability of the measurements should be known. Furthermore, knowledge on day-to-day repeatability of such measurements is important for longitudinal follow-up studies. But, there is a lack of data containing the information on within-session and between-session repeatability for VPT measurements at both glabrous and nonglabrous skin.

In this study, the baseline VPT at the nonglabrous site compared with the glabrous site was higher, which is in line with the previous research findings ${ }^{19}$. In clinical and research works, the common frequency investigated for the detection of the vibration perception is $125 \mathrm{~Hz}^{20)}$. In this study, we also used the test frequency of $125 \mathrm{~Hz}$ for the detection of VPT. In the glabrous skin, Merkel cells, Meissner's corpuscles, Ruffini corpuscles and Pacinian corpuscles are the mechanoreceptors responsible for the detection of vibrotactile inputs at different frequencies; such detection, on the other hand, results from the activity of the hair follicle afferent fibers, Ruffini corpuscles and Pacinian corpuscles in the human hairy skin ${ }^{20-23)}$. However, the Pacinian corpuscles mediate the detection of vibrotactile perception at high frequencies (more than 80 $\mathrm{Hz}{ }^{20)}$. Therefore, the detection of VPT in the current study was associated with the Pacinian corpuscles. 
According to the method of Bland and Altman (1986) ${ }^{17)}$, $95 \%$ of all differences from repeated measurements performed under the same circumstances would differ by a value less than the CoR, which is twice the standard deviation of the retest-test differences. On the other hand, ICC is a ratio of the between subjects variance to the total variance (between subjects + within subjects) ${ }^{24}$. As the value of ICC is closer to 1 , the repeatability is better. However, a value of ICC of 0.75 and above is considered to be indicative of a excellent repeatability and that of below 0.75 of poor to good reliability ${ }^{18)}$. In the current study, the CoR on the nonglabrous site was mostly twice as large as that on the glabrous side. Overall, relatively high ICC and low CoR values were found for VPT measurements at the glabrous finger skin (fingertip) indicating that the within-session and between-session measurements of VPT made with the tactile vibrometer are well repeatable. In contrast, VPT measurements at nonglabrous finger skin were found to be less repeatable as evidenced by the relatively low values of ICC with wider confidence intervals and accompanying high $\mathrm{CoR}$. It is not possible to compare the repeatability results of the current study with the previous research findings due to a lack of data reporting ICC and CoR values from both glabrous and nonglabrous skin. However, our findings at the glabrous skin are in line with the findings of Virokannas $(1992)^{25)}$, who reported a good repeatability for VPT measurements evaluated by repeated tests at the middle fingertip with an interval of at least 1 mo.

In view of the current study results, it is obvious that the glabrous skin is preferable while evaluating VPT. The findings of this study may guide the researchers to decide the measurement location while evaluating VPT. This is especially important in the context of evaluation of subjects with a history of exposure to hand-transmitted vibration.

In previous experiments of our laboratory using the similar experimental protocol, we demonstrated that exposure of healthy subjects to a relatively short duration of hand-arm vibration from grasping a vibrating handle significantly affected finger skin temperature and finger blood flow at both glabrous and nonglabrous skin. Hence, we wanted to investigate the response patterns occurring in cutaneous vibrotactile perception at glabrous and nonglabrous finger skin. In this study, a significant increase (in other words, reduction in cutaneous perception) in VPT was found under both $31.5 \mathrm{~Hz}$ and 250 $\mathrm{Hz}$ exposure conditions in the glabrous finger. On the other hand, a pronounced increase in VPT was revealed in the nonglabrous finger under the $250 \mathrm{~Hz}$ exposure condition. Such a significant change in VPT from acute exposure to vibration has also been reported by other researchers at the glabrous finger ${ }^{7,12}$. Our results provide evidence that acute vibration exposure at high frequency from grasping a handle may induce a significant increase in digital VPT at both glabrous and nonglabrous skin. In this study, overall, exposure to vibration at $250 \mathrm{~Hz}$ induced a larger increase in VPT. Our results are in line with the findings of Burström et al. (2009) ${ }^{12)}$, who reported that exposure to vibration at $31.5 \mathrm{~Hz}$ introduced a much lower temporary change in vibrotactile thresholds at the glabrous finger compared to a higher vibration stimulus of $125 \mathrm{~Hz}$. Our study findings also suggest that prior exposure of subjects to hand-arm vibration can influence their subsequent digital VPT responses. Considering such changes in VPT from prior exposure to vibration on the day of vibrotactile perception test, Burström et al. (2009) ${ }^{12)}$ suggested to follow the practice of avoiding exposure to vibration for $3 \mathrm{~h}$ prior to the examination of VPT among subjects working with hand-held vibrating tools, as recommended in the international Standard ISO 13091$1^{14)}$.

\section{Limitations}

There are several limitations to the current study. Firstly, this study involved healthy young subjects and thereby limiting the generalizability of the study results to other groups. Another limitation is the relatively small number of study subjects. Thirdly, the vibrometer used in this study allowed application of vibratory stimulus perpendicular to the skin surface from below. As the measurements in the glabrous and nonglabrous fingers were taken in pronated and supinated positions of the hand respectively, this difference could have affected the study results. Fourthly, VPT was measured at single stimulus frequency of $125 \mathrm{~Hz}$ which activates the Pacinian corpuscles. Therefore, caution should be exercised when extrapolating the data regarding other stimulus frequencies that activate Merkel cells (5 Hz), Meissner's corpuscles $(30 \mathrm{~Hz})$ on glabrous skin, and hair follicle receptors (5 and $30 \mathrm{~Hz}$ ) on nonglabrous skin ${ }^{22,23)}$. In this study, though the fingers were tested in the same order during the measurements, time effect during such measurements should be considered while interpreting the current results as measurements at each finger took some time and VPT after acute vibration exposure changes with time. Furthermore, glabrous and nonglabrous skin of all fingers were not evaluated in this study, however we believe that that the corresponding responses from fingers will be similar to the our study findings. In future, the results of the current study require statistical verification at other locations of glabrous and nonglabrous skin using a larger sample size.

\section{Conclusions}

Our results suggest that the quantifications of VPT made with the tactile vibrometer are repeatable, for both withinsession and between-session measurements, to reliably detect the changes in VPT at the glabrous finger skin. However, at the nonglabrous finger skin, the repeatability 
is limited. The findings also provide evidence that acute vibration exposure at high frequency from grasping a handle may induce significant increases in VPT at both glabrous and nonglabrous skin of fingers.

\section{References}

1) Sakakibara H, Hirata M, Toibana N. Impaired manual dexterity and neuromuscular dysfunction in patients with hand-arm vibration syndrome. Ind Health 2005; 43: 542-7.

2) Bovenzi M, Giannini F, Rossi S. Vibration-induced multifocal neuropathy in forestry workers: electrophysiological findings in relation to vibration exposure and finger circulation. Int Arch Occup Environ Health 2000; 73: 519-27.

3) Gerr F, Letz R. Vibrotactile threshold testing in occupational health: a review of current issues and limitations. Environ Res 1993; 60: 145-59.

4) Felix ER, Widerström-Noga EG. Reliability and validity of quantitative sensory testing in persons with spinal cord injury and neuropathic pain. J Rehabil Res Dev 2009; 46: 69-83.

5) Liu W, Lipsitz LA, Montero-Odasso M, Bean J, Kerrigan DC, Collins JJ. Noise-enhanced vibrotactile sensitivity in older adults, patients with stroke, and patients with diabetic neuropathy. Arch Phys Med Rehab 2002; 83: 171-6.

6) Deshpande N, Metter EJ, Ling S, Conwit R, Ferrucci L. Physiological correlates of age-related decline in vibrotactile sensitivity. Neurobiol Aging 2008; 29 : 765-73.

7) Harada N, Griffin MJ. Factors influencing vibration sense thresholds used to assess occupational exposures to hand transmitted vibration. Br J Ind Med 1991; 48: 185-92.

8) Harazin B, Harazin-Lechowska A. Effect of changes in finger skin temperature on vibrotactile perception threshold. Int J Occup Med Environ Health 2007; 20: 223-7.

9) Shun CT, Chang YC, Wu HP, et al. Skin denervation in type 2 diabetes: correlations with diabetic duration and functional impairments. Brain 2004; 127: 1593-605.

10) Duke J, McEvoy M, Sibbritt D, Guest M, Smith W, Attia J. Vibrotactile threshold measurement for detecting peripheral neuropathy: defining variability and a normal range for clinical and research use. Diabetologia 2007; 50: 2305-12.

11) Mahns DA, Perkins NM, Sahai V, Robinson L, Rowe MJ. Vibrotactile frequency discrimination in human hairy skin. J Neurophysiol 2006; 95: 1442-50.

12) Burström L, Lundström R, Hagberg M, Nilsson T. Vibrotactile perception and effects of short-term exposure to hand-arm vibration. Ann Occup Hyg 2009;
53: $539-47$

13) Nishiyama K, Watanabe S. Temporary threshold shift of vibratory sensation after clasping a vibrating handle. Int Arch Occup Environ Health 1981; 49: 21-33.

14) International Organization for Standardization. Mechanical vibration-vibrotactile perception thresholds for the assessment of nerve dysfunctionPart 1: Methods of measurement at the fingertips. ISO 13091-1, 2001. Geneva, Switzerland.

15) Mahbub MH, Inoue M, Yokoyama K, et al. Assessment of room temperature influence on finger blood flow response induced by short-term grasping of vibrating handle. Int Arch Occup Environ Health 2006; 79: 226.

16) Mahbub MH, Yokoyama K, Laskar MS, et al. Assessing the influence of antivibration glove on digital vascular responses to acute hand-arm vibration. J Occup Health 2007; 49: 165-71.

17) Bland JM, Altman DG. Statistical methods for assessing agreement between two methods of clinical measurement. Lancet 1986; 1: 307-10.

18) Fleiss JL. The design and analysis of clinical experiments. New York (USA): John Wiley and Sons; 1986.

19) Merzenich MM, Harrington TH. The sense of fluttervibration evoked by stimulation of the hairy skin of primates: comparison of human sensory capacity with the responses of mechanoreceptive afferents innervating the hairy skin of monkeys. Exp Brain Res 1969; 9: 236-60.

20) Dahlin LB, Thrainsdottir S, Cederlund R, et al. Vibrotactile sense in median and ulnar nerve innervated fingers of men with Type 2 diabetes, normal or impaired glucose tolerance. Diabet Med 2008; 25: 543-9.

21) Archer CB. Functions of the skin. In: T. Burns, S. Breathnach, N. Cox and C. Griffiths, editors, Rook's textbook of dermatology. 7 th edition. Vol. 1. Massachusetts (USA): Blackwell Science; 2004. p. 4.9-4.10.

22) Bolanowski SJ Jr, Gescheider GA, Verrillo RT, Checkosky CM. Four channels mediate the mechanical aspects of touch. J Acoust Soc Am 1988; 84: 1680 94.

23) Bolanowski SJ, Gescheider GA, Verrillo RT. Hairy skin: psychophysical channels and their physiological substrates. Somatosens Mot Res 1994; 11: 279-90.

24) Zwart JA, Sand T. Repeatability of dermatomal warm and cold sensory thresholds in patients with sciatica. Eur Spine J 2002; 11: 441-6.

25) Virokannas H. Vibration perception thresholds in workers exposed to vibration. Int Arch Occup Environ Health 1992; 64: 377-82. 